\title{
ON CONTINUITY IN TWO VARIABLES
}

\section{G. K. WILLIAMS ${ }^{1}$}

1. Introduction. It is well known that a real-valued function can be continuous in each fixed variable on the product of two intervals and yet fail to be continuous while, if a function is complex analytic in each fixed variable on the product of two open disks, then the function is complex analytic and hence continuous. The purpose of this note is to consider an intermediate case.

Let $f$ be a complex-valued function defined on a bicylinder $B$ $=B_{1} \times B_{2}$ with the property that $f(z, \cdot)$ is continuous for fixed $z \in B_{1}$ and $f(\cdot, w)$ is holomorphic for fixed $w \in B_{2}$. We shall say that $f \in H$ on $B$ if these conditions hold. In [3], we asked whether or not such a function $f$ must be continuous, thus, whether or not Hartogs' classical theorem could be generalized to this extent. In this note we shall give an example to show that $f$ need not be continuous and we also give some conditions which are sufficient to insure the continuity of $f$.

2. Counterexample. Before exhibiting the example we need the following result.

Lemma. Let $f \in H$ on $B=B_{1} \times B_{2}$. Then $f$ is continuous on $B$ if and only if each sequence of functions $\left\{f\left(\cdot, w_{n}\right)\right\}$, where lim $w_{n}=w_{0} \in B_{2}$, converges almost uniformly on $B_{1}$.

Proof. "if." This follows from the equivalence of continuous convergence and uniform convergence. See [1].

"only if." This follows from uniform continuity.

Now let $B$ be the unit bicylinder, $B=\{(z, w):|z|<1,|w|<1\}$. Using Runge's theorem there exists a sequence of polynomials $\left\{P_{n}(z)\right\}$ which converge to zero on $|z|<1$ but the convergence is not uniform on any neighborhood of any point of the real axis in $|z|<1$. Using this sequence we define a function $F$ on $B$ as follows:

(i) on the circles in $|w|<1$ of radius $1 / n, n=2,3, \cdots$, let $F(z, w)$ $=P_{n}(z)$. Let $F(z, 0) \equiv 0$.

(ii) For $w$, with $1 /(n+1)<|w|<1 / n, n=1,2, \cdots$, consider the circles $C_{t}$ with radius $r=t / n+(1-t) /(n+1), 0<t<1$. For $w \in C_{t}$ define

$$
F(z, w)=t P_{n}(z)+(1-t) P_{n+1}(z) .
$$

Received by the editors April 24, 1969.

1 This work was partially supported by NSF GP-7265. 
Then $F \in H$ on $B$ but is not continuous by virtue of the lemma.

3. Another characterization. Let $F \in H$ on the unit bicylinder. Define the "radius of continuity" as follows. For $z_{0}$, with $\left|z_{0}\right|<1$, $R\left(z_{0}\right)$ is the distance from $w=0$ to the nearest point $w_{0}$ such that $f$ is discontinuous at $\left(z_{0}, w_{0}\right)$. Using a method due to Rothstein [2] we shall prove the following theorem.

Theorem. Let $f \in H$ on $B$, where $B$ is the unit bicylinder. Then $f$ is continuous if and only if $R$ is superharmonic.

For the proof we shall need the following results.

Proposition 1. Let $S$ be a complete metric space and $F$ a family of continuous real-valued functions on $S$. Then, if for each $\chi \in S$, the set $\{f(\chi): f \in F\}$ is bounded, there exists a closed sphere $U$ of positive radius in $S$ and a number $M$ such that $|f(\chi)| \leqq M$ for all $f \in F$ and all $\chi \in U$.

Proof. This is well known.

Proposition 2. Let $h$ be superharmonic in $|z|<1$ with $h \geqq 0$. Let $S_{n}, S, T_{n}$ be points in $|z|<\alpha<1$ with $S_{n} \rightarrow S$ and $\left|T_{n}-S\right|>d>0$. Suppose $J_{n}$ is a Jordan arc joining $S_{n}$ to $T_{n}$ in $|z|<\alpha$. Then if $h(z) \geqq r$ on all $J_{n}$, we have $h(S) \geqq r$.

Proof. The proof is in [2].

Now let $D(k)$ denote a domain in $|z|<1$ for which $R(z) \geqq k$ and which is maximal with respect to this property.

Lemma 1. Given $k$ such that $0<k<1$, there exists a $D(k)$.

Proof. We need only show that there exists an open disk $D$ contained in $|z|<1$ such that $f$ is continuous on $D \times\{|w| \leqq k\}$.

To see this we apply Proposition 1 to the family $\left\{g_{v}(z)\right\}$, where

$$
g_{w}(z)=|f(z, w)|, \quad|z| \leqq 1 / 2, \quad|w|<k^{\prime}, \quad k<k^{\prime}<1 .
$$

Hence there exists an open disk $D$ in $|z|<1 / 2$ and a constant $K$ such that $|f| \leqq K$ on $D \times\left\{|w|<k^{\prime}\right\}$. Then using Vitali's theorem and the lemma of $\S 2$ we get our result.

Note that if $f \in H$ on $B$ and is locally bounded, then $f$ is continuous.

Leмma 2. If $q$ is a boundary point of $D(k)$ and $|q|<1$, then there exists a sequence $q_{n} \rightarrow q$ with $R\left(q_{n}\right)<k$.

Proof. Clear.

Lemma 3. Let $\left\{D_{n}(k)\right\}$ be a sequence of domains of type $D(k)$. Let $z_{0},\left|z_{0}\right|<1$, be a limit point of the $D_{n}(k)$ and let $d_{n}$ be the diameter of $D_{n}(k)$. If $R\left(z_{0}\right)<k$, then $\lim d_{n}=0$. 
Proof. Apply Proposition 2.

Proof of THE THEOREM. The "only if" part of the theorem is trivial. For the "if" part, let $k$ be such that $0<k<1$. We know by Lemma 1 that there exists a $D(k)$ in $|z|<1$. Suppose this $D(k)$ has a boundary point $z_{1}$, with $\left|z_{1}\right|<1$. Let $K_{1}$ be a closed disk in $|z|<1$ with center $z_{1}$ and let $M$ be the set of all boundary points $q$ of all domains of type $D(k)$, where $q \in$ int $K_{1}$, and let $\bar{M}$ be the closure of this set. Using Proposition 1 on $\bar{M} \cap K_{1}$ there exists a closed disk $K_{2} \subset K_{1}$ with center $z_{2} \in \bar{M}$ and a number $m>0$ such that $|f(z, w)| \leqq m$ for $z \in \bar{M} \cap K_{2}$ and $|w|<k$.

By Lemma 2 , there exists in $K_{2}$ a point $z_{3}$ with $z_{3} \in$ int $K_{2}$ and $R\left(z_{3}\right)=k_{1}<k$. Now let $d>0$ be chosen small enough that the boundaries of all $D(k)$ which have points in common with $K_{3}=\left\{\left|z-z_{3}\right| \leqq d\right\}$ are completely contained in $\bar{M} \cap K_{2}$. This is possible by Lemma 3 . Then by the maximum principle we have $|f(c, w)| \leqq m$ for $|w|<k$ and all $c \in D^{\prime}(k)$ where $D^{\prime}(k)$ is any domain of type $D(k)$ which intersects $K_{3}$. But using Proposition 1 we see that these intersections must be dense in $K_{3}$ and hence $f$ is bounded and thus is continuous on int $K_{3} \times\{|w|<k\}$. This contradicts the fact that $R\left(z_{3}\right)=k_{1}<k$ and proves the result.

4. Acknowledgement. The author is indebted to Professor W. Stoll for suggestions concerning the counterexample.

\section{REFERENCES}

1. C. Caratheodory, Theory of functions, Chelsea, New York, 1954.

2. W. Rothstein, Ein neuer Beweis des Hartogschen Hauptsatzes und seine Ausdehnung auf mermorphe Funktionen, Math. Z. 53 (1950), 84-95.

3. G. K. Williams, Non-analytic functions of two complex variables, Duke Math. J. 34 (1967), 249-254.

Southwestern at Memphis 